Review Article

\title{
Prospects for Malaria Vaccines: Pre-Erythrocytic Stages, Blood Stages, and Transmission-Blocking Stages
}

\author{
Jingtong Zheng (D), He Pan (D), Yinuo Gu, Xu Zuo (D), Nan Ran (D), Yuze Yuan (D), \\ Chao Zhang $\mathbb{D}$, and Fang Wang $\mathbb{D}$ \\ Department of Pathogenobiology, College of Basic Medicine, Jilin University, Changchun 130000, China \\ Correspondence should be addressed to Fang Wang; wf@jlu.edu.cn
}

Received 23 May 2019; Revised 6 August 2019; Accepted 9 September 2019; Published 3 October 2019

Academic Editor: Aparup Das

Copyright (C) 2019 Jingtong Zheng et al. This is an open access article distributed under the Creative Commons Attribution License, which permits unrestricted use, distribution, and reproduction in any medium, provided the original work is properly cited.

\begin{abstract}
Malaria is a disease of public health importance in many parts of the world. Currently, there is no effective way to eradicate malaria, so developing safe, efficient, and cost-effective vaccines against this disease remains an important goal. Current research on malaria vaccines is focused on developing vaccines against pre-erythrocytic stage parasites and blood-stage parasites or on developing a transmission-blocking vaccine. Here, we briefly describe the progress made towards a vaccine against Plasmodium falciparum, the most pathogenic of the malaria parasite species to infect humans.
\end{abstract}

\section{Introduction}

Malaria caused by Plasmodium falciparum $(P f)$ is a parasitic disease whose pathology seriously endangers human health. In 2017, 219 million people were infected with this parasite species and about 435,000 people, mostly children under the age of 5 years, died from infections with it (Figure 1).

In 2015, the World Health Organization (WHO) endorsed a new Global Technical Strategy for Malaria (http:// www.who.int/). This strategy includes ambitious goals for malaria control and elimination over the next 15 years. A key target is the elimination of malaria in at least 10 countries by 2020. The WHO has recently reported on the progress made and challenges outstanding in 21 countries (called the E-2020 countries), which identified it in 2016 as having the potential to become malaria free by 2020 (http://www.who.int/). Although remarkable achievements in malaria control have been made in recent years, serious difficulties persist because of the emergence and spread of parasite resistance to antimalarial drugs [1]. Moreover, development of resistance to pyrethroid insecticides has enabled the mosquito vectors of malaria to survive when they make contact with long-lasting insecticide-treated mosquito nets [2]. The development of a safe, inexpensive, and effective vaccine is widely believed to be critical for the control and eradication of malaria. The WHO, United Nations Development Program, and the World Bank have identified malaria vaccine research as one of the top three global priorities for vaccine development. Much research effort has been directed at the following four different approaches towards a malaria vaccine: a vaccine targeting the whole organism, a live attenuated vaccine, a genetically engineered vaccine, and a subunit vaccine, but no widely accepted vaccine has been developed to date. Recently, some studies investigating the effects of Pf sporozoite (SPZ) vaccines have reported that they hold great promise for protection in humans, but larger sample sizes are needed to validate their protective effects $[3,4]$. Researchers face challenges increasing vaccine antigenicity while addressing safety issue as well.

According to the life history of $P f$ in humans, malaria vaccines can be divided into three types (Table 1): preerythrocytic, blood-stage, and transmission-blocking vaccines (TBVs). Vaccine candidates are being tested in clinical trials where several have shown potential. Here, we briefly introduce the progress made in the development of a vaccine against $P f$ and the current advances being made in this field. 


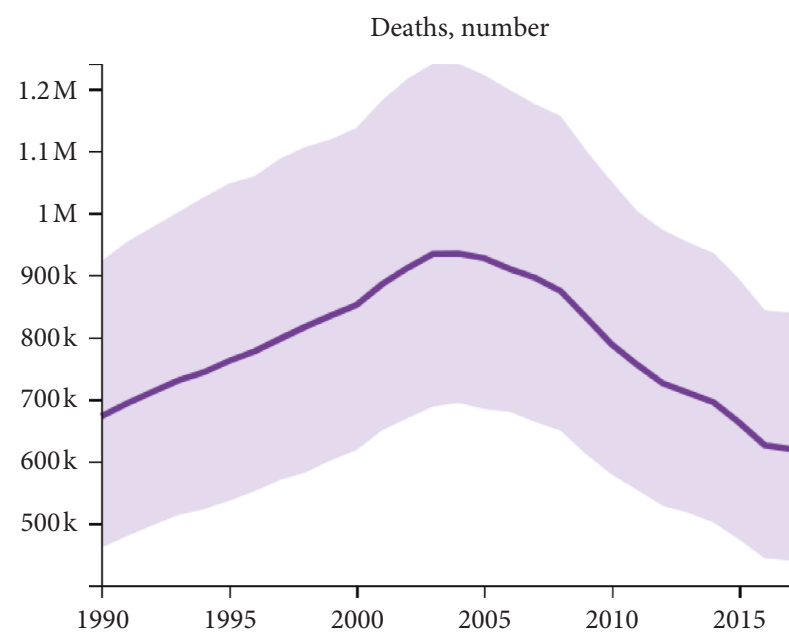

(a)

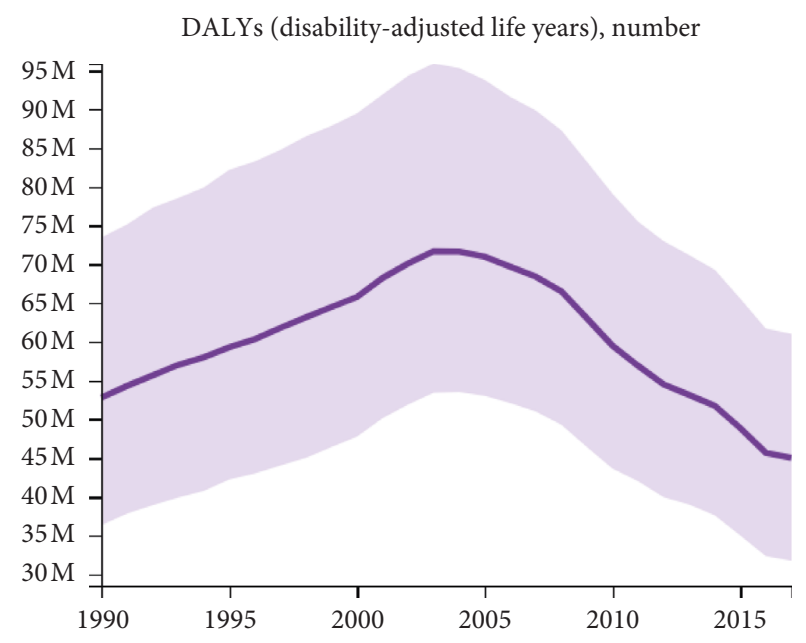

(b)

FIGURE 1: Deaths of malaria (a) and DALYs of malaria (b) between 1990 and 2017, all ages (created with data from the Global Burden of Disease Study 2017 (GBD 2017) results).

\section{Pre-Erythrocytic Vaccines}

Pre-erythrocytic vaccines, also known as anti-infective vaccines, mainly target the spores of $P f$. They induce specific antibodies that kill infected liver cells or interfere with the malaria parasite during liver cell proliferation, thereby blocking the release of infectious merozoites and achieving an anti-infection effect (Figure 2(a)). Vaccines targeting preerythrocytic Plasmodium must have a $100 \%$ protection rate to achieve a real protective effect. At present, most preerythrocytic malaria vaccine research is focused on the development of subunit vaccines against parasite proteins such as the Pf circumsporozoite protein (PfCSP), the thrombospondin-related adhesion protein (TRAP), and the liver stage antigen (LSA).

2.1. PfCSP Vaccines. Located on the surface of the mature sporozoite as a $40-60 \mathrm{kDa}$ pre-erythroid antigen, PfCSP plays a key role in sporozoite invasion of liver cells [5]. Till now, DNA vaccine against CSP has been studied for years, which is considered to be a simple and stable vaccine, but the situation of the DNA vaccine in the human body is still in the stage of development and testing. Therefore, in order to better induce an effective immune response, the DNA vaccine gp96NTD-CSP was designed. Heat-shock protein (HSP) is designed to induce dendritic cell maturation and promote cross-antigen presentation, making it is an important immune adjuvant and immune delivery system. gp96 of HSP can be effectively presented to major histocompatibility complex I, leading to $\mathrm{CD}^{+} \mathrm{T}$-cell activation. Tan et al. [6] reported that the adjuvant properties of gp96NTD enhanced the immunogenicity and protective efficacy of this vaccine by inducing high levels of CSPspecific antibodies and a strong $\mathrm{CD}^{+} \mathrm{T}$-cell response. It is reported that CSP-based structures induce high levels of protection in mice, but they are less immunogenic in humans. The reason may be the lack of antibodies caused by
DNA vaccine itself or the inability of T-cell immune response to completely eliminate parasites.

2.1.1. RTS,S/AS01 and RTS,S/AS02. RTS,S/AS01 is a current lead recombinant candidate vaccine against malaria [30]. RTS,S is the vaccine with epitopes $\mathrm{R}$ and T of $\mathrm{B}$ and T cells recombined to the C-terminal end of CSP of PF 3D7, membrane surface protein $(S)$ of hepatitis virus $C$, and free copy of protein S. AS01 is the adjuvant system to increase the immune system response. Protective immune responses after vaccination with RTS,S are dependent primarily on antibody responses against the central repeat region [31-33]. The RTS,S/AS01 vaccine, which was found to protect African children against clinical and severe malaria [7], has been tested in a phase III trial [8] and has received a positive rating from the European Medicines Agency, but it may not be effective against $P f$ isolates from southern and central Africa transmission-blocking vaccines or other global regions. Since the C-terminal region contains some important T-cell epitopes [9] which related to $\mathrm{CD} 4^{+} \mathrm{T}$-cell responses, the high genetic diversity in the C-terminal region of the PfCSP molecule may lead to the lack of overall protective efficacy. Therefore, genetic diversity assessment of the C-terminal region of PfCSP is an important aspect of developing an RTS,S/AS01 vaccine for widespread use. Moreover, a phase III trial indicates that the C-terminal sequence-unmatched strains show lower efficacy [10]. To evaluate the clinical malaria incidences and serious adverse events, 3084 children (aged 3-7 years) were enrolled in the phase III trial for 3-year studies. In the studies, primary outcome is the occurrence of severe malaria meeting the primary case definition, and secondary outcome includes the occurrence of clinical malaria meeting the primary and secondary case definitions, occurrence of malaria hospitalization meeting each of the case definitions, prevalence of anemia, and prevalence of parasitemia. It is reported that 66 of the severe malaria cases were reported in 3-year studies. Moreover, clinical malaria 
TABLE 1: List of Plasmodium vaccines in pre-erythrocytic stages, blood stages, and transmission-blocking stages.

\begin{tabular}{|c|c|c|c|c|c|}
\hline $\begin{array}{l}\text { Vaccine } \\
\text { group }\end{array}$ & $\begin{array}{l}\text { Vaccine } \\
\text { name }\end{array}$ & $\begin{array}{c}\text { Vaccine } \\
\text { type }\end{array}$ & $\begin{array}{c}\text { Malarial antigen } \\
\text { targeted }\end{array}$ & $\begin{array}{l}\text { Mechanism of } \\
\text { action }\end{array}$ & References \\
\hline \multirow{6}{*}{$\begin{array}{l}\text { Pre-erythrocytic } \\
\text { vaccines }\end{array}$} & PfCSP vaccines & & PfCSP & $\begin{array}{l}\text { Antibodies to PfCSP block the } \\
\text { sporozoite invasion of liver cells }\end{array}$ & {$[5,6]$} \\
\hline & $\begin{array}{l}\text { RTS,S/AS01 and } \\
\text { RTS,S/AS02 }\end{array}$ & $\begin{array}{l}\text { Subunit } \\
\text { vaccines }\end{array}$ & $\begin{array}{c}\text { Hepatitis B surface antigen and the } \\
\text { central repeat and C-terminal regions } \\
\text { of CSP }\end{array}$ & $\begin{array}{l}\text { Protective immune responses after } \\
\text { vaccination with RTS,S are dependent } \\
\text { primarily on antibody responses } \\
\text { against the central repeat region }\end{array}$ & {$[7-11]$} \\
\hline & $\begin{array}{l}\text { PfCSP bacteria } \\
\text { vaccines }\end{array}$ & & PfCSP & $\begin{array}{l}\text { Bacteria need assistance to stimulate } \\
\text { the innate immune system }\end{array}$ & {$[12,13]$} \\
\hline & TRAP vaccines & $\begin{array}{l}\text { Subunit } \\
\text { vaccines }\end{array}$ & SSP-2 & $\begin{array}{c}\text { Antibodies to SSP-2 block the invasion } \\
\text { of mosquito salivary glands and } \\
\text { hepatocytes }\end{array}$ & {$[14]$} \\
\hline & LSA vaccines & $\begin{array}{l}\text { Subunit } \\
\text { vaccines }\end{array}$ & LSA-1/LSA-3 & $\begin{array}{l}\text { Elicit a pre-erythrocyte antigen } \\
\text { response in the majority of individuals } \\
\text { from different age groups }\end{array}$ & {$[15-18]$} \\
\hline & PfSPZ vaccines & $\begin{array}{l}\text { Live attenuated } \\
\text { vaccines }\end{array}$ & PfSPZ & $\begin{array}{c}\text { Antibodies to PfSPZ block the parasite } \\
\text { arrival to the liver or during their } \\
\text { development in this organ }\end{array}$ & [3] \\
\hline \multirow{3}{*}{$\begin{array}{l}\text { Asexual blood-stage } \\
\text { vaccines }\end{array}$} & MSP1 vaccines & $\begin{array}{l}\text { Subunit } \\
\text { vaccines }\end{array}$ & $\mathrm{MSP}_{42} / \mathrm{MSP}_{38} / \mathrm{MSP} 1_{83}$ & $\begin{array}{l}\text { Antibodies to MSP1 to block the } \\
\text { parasite invasion of erythrocyte }\end{array}$ & [19] \\
\hline & AMA-1 vaccines & $\begin{array}{c}\text { Subunit } \\
\text { vaccines }\end{array}$ & AMA-1 & $\begin{array}{l}\text { AMA-1 plays an essential role in } \\
\text { parasite survival. Antibodies to AMA-1 } \\
\text { may kill the parasite }\end{array}$ & {$[20-22]$} \\
\hline & Rh5 vaccines & $\begin{array}{l}\text { Subunit } \\
\text { vaccines }\end{array}$ & Rh5 & $\begin{array}{l}\text { Antibodies to Rh5 block the parasite } \\
\text { invasion of erythrocyte by forming } \\
\text { complex with cyrpa and ripr }\end{array}$ & {$[23-25]$} \\
\hline \multirow{2}{*}{$\begin{array}{l}\text { Transmission- } \\
\text { blocking vaccines }\end{array}$} & Pfs 25 vaccines & $\begin{array}{l}\text { Subunit } \\
\text { vaccines }\end{array}$ & Pfs 25 & $\begin{array}{l}\text { Pfs } 25 \text { is the target on which the parasite } \\
\text { survives and interacts with the } \\
\text { mosquito midgut. Antibodies to Pfs } 25 \\
\text { control the transmission of malaria } \\
\text { parasites from human hosts to the } \\
\text { mosquito vectors }\end{array}$ & {$[26-28]$} \\
\hline & Pfs $48 / 45$ vaccines & $\begin{array}{l}\text { Subunit } \\
\text { vaccines }\end{array}$ & Pfs $48 / 45$ C-terminus & $\begin{array}{l}\text { Pfs } 48 / 45 \text { is the target on which male } \\
\text { gamete attaches to female gamete. } \\
\text { Antibodies to Pfs } 48 / 45 \text { can induce } \\
\text { transmission-blocking antibody } \\
\text { response during infection }\end{array}$ & [29] \\
\hline
\end{tabular}

incidences in the four-dose, three-dose, and control groups were 1.079 PPY, 1.108 PPY, and 1.016 PPY, respectively [11].

Another candidate vaccine was that RTS,S combined with adjuvant systems AS02 named RTS,S/AS02 [30]. It is reported that both RTS,S/AS01 and RTS,S/AS02 vaccines exhibited better CS-specific immune responses than nonadjuvanted RTS,S. But the anti-CS antibody response to RTS,S/AS01 was much better than RTS,S/AS02 in studies in animals and phase IIa CHMI trial conducted at WRAIR, which might be one of the reasons that RTS,S/AS01 was much widely used than RTS,S/AS02.

2.1.2. PfCSP Bacteria Vaccines. In addition, vectors like viruses and bacteria can be also used as malaria vaccines. Because these vectors are based on the whole organism, they usually do not need assistance to stimulate the innate immune system compared with DNA vaccines. Bacterial vectors are one of the most common delivery vectors for malaria vaccines. Salmonella enterica serovar Typhi live vector vaccines have been used widely since 2009 [12]; in 1997, Gonzalez et al. [13] inserted the PfCSP gene into the pUC19 vector to create pUC-PfCSP. The construct was recombined with pKK233-3 to obtain pKK233-PfCSP and then transformed into the S. enterica serovar Typhi strain CVD 908 by electroporation. The resultant CVD 908 omega strain (delta aroC1019::tacP-rcsp) was well tolerated by 10 volunteers who each received two doses of $5 \times 10^{7}$ organisms 8 days apart. The volunteers showed a fourfold rise in their antibody levels against a recombinant portion of CSP, and CSP-specific $\mathrm{CD}^{+}$cytotoxic $\mathrm{T}$ lymphocyte activity was induced [13].

2.2. TRAP Vaccines. TRAP, also known as the PfSPZ surface protein 2 (SSP-2), is a major antigen that plays an important role in SPZ invasion of mosquito salivary glands and hepatocytes [14]. Recently, researchers have found that TRAP interacts directly with integrins on the surfaces of human cells [34]. The multiepitope (ME) string TRAP antigen contains a fusion protein of $\mathrm{ME}$, followed by pre-erythrocytic TRAP from the T9/96 Pf strain [35]. Except choosing the antigen, to be a candidate vaccine against malaria, inducing strong T-cell and B-cell responses is also important.

Vaccine using recombinant virus like influenza viruses, poxvirus, syndicate virus, yellow fever virus, adenovirus, and human cytomegalovirus has showed good effects in animal models [36]. Adenovirus-based vaccines were originally 
developed to induce strong $\mathrm{T}$-cell and $\mathrm{B}$-cell responses, which has an effort like intrinsic adjuvant to eliminate the need for chemical adjuvants in DNA vaccines. However, there is still a big difference between animal tests and human tests. The current method is to combine the vector with the chemical adjuvant. Studies have shown that the titre of antibody induced by adjuvant vaccine is significantly higher than that of the control group without immune adjuvant. Therefore, the rational use of chemical adjuvants will be conducive to the induction of protective T-cell immune response.

Studies showed that prime-boost regimens using the simian adenovirus 63- (ChAd63-) modified vaccinia virus Ankara (MVA) vector expressing the clinically relevant $P f$ ME-TRAP antigen have shown outstanding protective efficacy in mice and macaques [34]. Heterologous prime boosting with ChAd63-MVA has been shown to be a potent inducer of $\mathrm{T}$ cells in humans and has demonstrable efficacy when expressed with the pre-erythrocytic ME-TRAP antigen insert [37]. This antigenic insert remains the most promising for a vectored liver-stage vaccine [38]. It is reported that after giving ChAd63 MVA ME-TRAP vaccine, anti-TRAP IFN- $\gamma$ responses in infants were as high as that in adults. IgG responses, mainly composed of IgG1 and IgG3 isotypes, were higher than those in other time after boosting in the vaccines.

Moreover, Gomez et al. [39] inserted the SSP-2 gene into pBSK to obtain pBSK-SSP-2 and recombined it with pMOhyl1 to obtain pMO-SSP-2. After transformation by electroporation into the SL3261 strain, positive strains were screened and cultured. In mice immunized intranasally, serovar Typhimurium constructs secreting SSP-2 stimulated interferon-gamma splenocyte responses [39].

2.3. LSA Vaccines. LSA-1 is a $230 \mathrm{kDa}$ protein with a central region containing 86 repeats of the 17-amino-acid sequence EQQSDLEQERLAKEKLQ or minor variations thereof [40]. As the LSA-1 immune response is close to that of naturally transmitted parasites, this antigen has become an attractive vaccine candidate $[15,16]$, and several clinical trials have tested the effects of LSA-1 vaccines. LSA-NRC, an antigen containing $\mathrm{T}$-cell epitopes from the $\mathrm{N}$ and $\mathrm{C}$ terminal regions of LSA-1 and several central amino acid repeats, was expressed in Escherichia coli and combined with AS01B or AS02A to induce LSA-1-specific cellular immune responses [16]. In addition to LSA-1, the highly conserved LSA-3 protein is a candidate for a subunit vaccine [17]. A vaccine containing the LSA-3 protein elicited a pre-erythrocyte antigen response in the majority of individuals from different age groups [18]. However, its safety, immunogenicity, and efficacy have not been reported on to date [16].

2.4. PfSPZ Vaccines. Plasmodium SPZs are transmitted to and multiply within the human liver after being carried in the bloodstream when an individual is bitten by a female Anopheles mosquito [36]. Therefore, pre-erythrocytic vaccines against malaria need to be highly effective at killing liver SPZs because as little as one SPZ can initiate a malaria infection. For a PfSPZ vaccine to work effectively, it needs to kill SPZs before they arrive at the liver or during their development in this organ. PfSPZ vaccines can be divided into several groups. Attenuated PfSPZ vaccine is the whole parasite attenuated by irradiation. It is reported that sporozoite vaccine can induce $\mathrm{T}$ cell against the malaria infection. Moreover, since the expression of IFN- $\gamma$ was related to $\mathrm{CD}^{+} \mathrm{T}$ cells, animal studies demonstrated that the vaccine induced high level of PfSPZ-specific $\mathrm{CD}^{+} \mathrm{T}$ cells and IFN- $\gamma$. Compared with heat-inactivated PfSPZ, the frequency of IFN- $\gamma$-producing CD8+ T cells was much higher in attenuated parasite vaccine. Till now, one of the highest levels (more than 90\%) of protection against malaria in humans has been achieved only by immunization with radiation-attenuated PfSPZ. The PfSPZ-chemoprophylaxis vaccine (PfSPZ-CVac) is attenuated by concomitant administration of chemoprophylaxis [3]. It is reported that PfSPZ-CVac achieves similar protective efficacy but with fewer parasites. It is interesting that only $22 \%$ ( 2 of 9 ) protected volunteers had been induced IFN- $\gamma$-producing $\mathrm{CD}^{+} \mathrm{T}$ cells at the time of CHMI, which is much lower than radiation-attenuated PfSPZ. The difference should be carefully concerned by future studies. Except attenuated by irradiation and using antimalarial drug, gene knockout is another way to make the PfSPZ vaccine. One of the lead candidates is PfSPZ-GA1. This vaccine is a kind of parasite in which the two proteins Pfb9 and Pfslarp were knocked out, which allows liver infection of the parasite without subsequent development in the liver.

Despite the difficulty of this task, one study was successful at achieving 100\% protection (in 6 out of 6 subjects) with a radiation-attenuated PfSPZ vaccine [41]. Elsewhere, three doses of a chemoattenuated PfSP vaccine prevented infection in 9 out of $9(100 \%)$ volunteers [3]. These results show that attenuated PfSPZ vaccines show encouraging evidence of efficacy in clinical trials. Till now, the trials of PfSPZ vaccine in Mali confirm efficacy against naturally transmitted parasites, but the dosage, regimen, and route of administration of vaccine are also need to be analyzed. It is shown that PfSPZ vaccine can protect against a heterologous CHMI (3 weeks and 24 weeks) by a 3-dose regimen. Moreover, PfSPZ vaccine was given to 41 adults in Mali. It is reported that $66 \%$ of participants were infected, compared with $93 \%$ of the control group after five-dose vaccines [42]. With low rate of systemic adverse event, the vaccine was shown to be well tolerated and safe. However, the level of CD4, CD8, and $\gamma \delta$ T cells showed no difference between the groups, which was different from other studies. The difference in infection rate and protection effect may be due to their regional differences. The infection rate in epidemic areas should be significantly higher than that in nonepidemic areas. Till now, it is easier to make a vaccine that can safely protect short-term travellers. Keeping antibody levels and specific $\mathrm{T}$-cell responses is still a challenge for the development of PfSPZ vaccine.

\section{Asexual Blood-Stage Vaccines}

Clinical signs and symptoms, including recurrent fever, can emerge after Plasmodium parasites circulate as blood-stage 


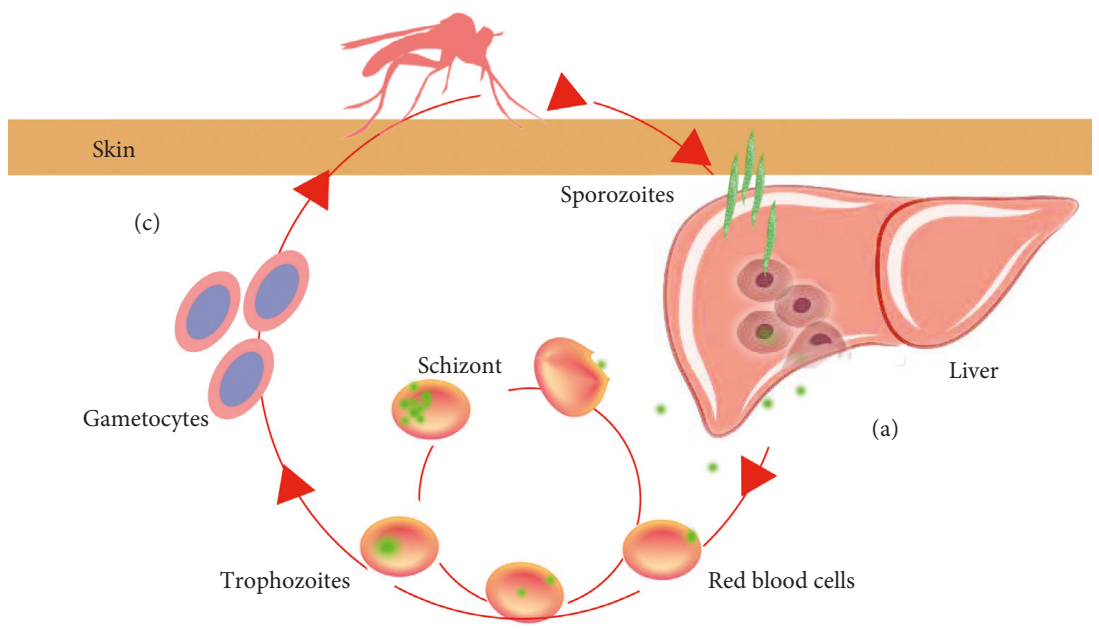

(b)

Figure 2: Vaccines target the life cycle of Plasmodium falciparum. (a) Pre-erythrocytic Plasmodium vaccine: vaccine produces antibodies that kill infected liver cells or interfere with the malaria parasite during liver cell proliferation; (b) asexual blood-stage vaccine: vaccine aims primarily at reducing parasite load or eliminating circulating parasites; (c) vaccine aims at controlling the transmission of malaria parasites from human hosts to the mosquito vectors.

parasites in the red blood cells of the human body (Figure 2(b)). The presence of these stages (merozoites, rings, trophozoites, schizonts, and gametocytes) causes severe pathology and even death. After entering the human red blood cells from the liver, the surface protein of the $P f$ merozoite can remain in the red blood cell membranes and be exposed directly to the host's immune system. This can stimulate T cells and B cells to produce immune responses. It is reported that $\gamma \delta$ T cells may produce antibody and perform protective immunity to blood stage of malaria infection. The study has shown that when infected with parasite, IFN- $\gamma$ and IgG2a in $\gamma \delta$ T-cell-depleted mice were significantly lower than in control mice [43]. Therefore, research on blood-stage malaria vaccines has for a long time been a "hot spot" of vaccine research.

3.1. Merozoite Surface Protein 1 (MSP1) Vaccines. MSP1 is located on the merozoite surface where it plays a key role in erythrocyte invasion. PfMSP1, which is a blood-stage antigen, is hydrolyzed into $\operatorname{PfMSP} 1_{83}, \operatorname{PfMSP} 1_{28}, \operatorname{PfMSP} 1_{38}$, and $\mathrm{PfMSP}_{42}$ before merozoite invasion of the erythrocytes. During invasion, $\mathrm{PfMSP}_{42}$ is processed into $\mathrm{MSP}_{33}$ and $\mathrm{MSP}_{19}$ [19]. PfMSP1 $1_{19}$ contains the structural domain of the epidermal growth factor and is rich in cysteine residues. It can become anchored to the merozoite surface and combine with the erythrocyte surface receptor band 3 protein. In trials conducted to test PfMSP1-based vaccines, all the candidate vaccines were found to be safe and highly immunogenic [44]. Some reports have indicated that MSP $_{19}$ has no efficacy in terms of reducing the parasite multiplication rate in homologous controlled human malaria infections or natural infections [20, 45, 46]. Alaro et al. [21] produced recombinant $P f M S P 1_{19}$ fused to the
$\mathrm{N}$-terminus of the $P f$ merozoite surface protein 8 with the low-complexity Asn/Asp-rich domain (rPfMSP8) lacking. Immunization of mice with the chimeric $\mathrm{rPfMSP} 1 / 8$ vaccine elicited strong T-cell responses to the conserved epitopes associated with the rPfMSP8 fusion partner. PfMSP1/8specific rabbit IgG was shown to potently inhibit the in vitro growth of FVO and 3D7 strains of blood-stage Pf parasites [22]. Since people can be infected with different kinds of parasites in different regions, a wide range of epidemiological surveys is also needed. By analyzing the distribution of certain strains in malarial regions and the changes over time, it can help people develop specific vaccines for specific areas (although the vaccine may not be effective elsewhere). It is reported that $\mathrm{MSP}_{19}$ in FVO or FuP strain vaccines may be more effective than 3D7 strain in Malian because the most common parasites strain causing malaria are from FVO or FuP [47]. Moreover, some kinds of bioinformatics software like BioStructMap can be used in the research screening. By studying the selection in different regions and the diversity of structural patterns, the bioinformatics software can be used to analyze the good $\mathrm{MSP}_{19}$ vaccine candidates of the parasite.

3.2. Apical Membrane Antigen 1 (AMA-1) Vaccines. AMA-1 is expressed in the sporozoite, hepatic, and erythrocytic stages [23] where it plays an essential role in parasite survival [45]. The AMA-1/AS02 blood-stage vaccine demonstrated no overall efficacy, but sieve analysis revealed allele-specific efficacy against the vaccine strain, suggesting that AMA-1 could be a useful inclusion in a multicomponent vaccine [46]. In a series of trials, experimental malaria vaccines formulated with AMA-1 and an AS01B or AS02A adjuvant system had demonstrable safety, tolerability, and 
immunogenicity [20-22]. The AMA-1 molecule has 16 conservative cysteine residues, which divides the protein into three substructures. Among them, AMA-1 (III), which is located in the C-terminal region, is genetically conserved and has been considered as a candidate vaccine component. Takala et al. [47] found that $P f$ chimeric protein 2.9 (PfCP2.9), which consists of MSP $1_{19}$ and AMA-1 (III) sequences, induced parasite-inhibitory antibodies in rabbits and monkeys. The safety and immunogenicity of PfCP-2.9 formulated with the novel Montanide ISA 720 adjuvant were tested, and all the candidate vaccines were shown to be safe and immunogenic [47].

The development of malaria vaccine is a long and arduous process because people can be infected with different kinds of malaria strains. Considering the complexity of malaria antigens, if the parasite proteins obtained from people are different from the ones used for vaccination, it may lead to the immune response that may be less effective or even ineffective. Therefore, an ideal candidate vaccine should consider the effect of alleles on immunogenicity. A method to solve the problem is that AMA-1-C1 (a mixture of AMA-1-FVO and AMA-1-3D7 recombinant proteins) is designed to protect against a majority of parasite genotypes. But the effective titres of antibodies to the proteins and the integrity of the protein by long-term storage are also need to be concerned. Now, some research studies try to generate a vaccine that targets the conserved regions of the AMA-1, but such a vaccine is not used in clinical till now.

3.3. Rh5 Vaccines. The blood stages of a malaria infection cause morbidity and mortality in humans. Hence, vaccines targeting these stages aim at protecting the human host against clinical disease and death. Till now, extensive efforts usually focused on a small number of well-studied merozoite antigens targeting naturally acquired immunity to induce antibodies that block erythrocyte invasion [36]. Rh5, as the most important candidate for the blood-stage vaccine, rapidly invades red blood cells by forming complex with cyrpa and ripr and binding with basigin of red blood cells. Therefore, Rh5-targeted vaccine can prevent pathogens from invading the human body. At present, the 3D image information of the protein complexes has been studied. Structural analysis of the complexes will contribute to screen the target position of complexes and development of the vaccines. In addition, studies have found that p113 can interact with Rh5nt directly, so it also can be developed as a candidate vaccine [23]. Moreover, a group of human monoclonal antibodies (mabs) against pfrh5 were isolated from the peripheral blood B cells of the volunteer. One subgroup of mabs neutralizes the antibody while the other subgroup of monoclonal antibodies plays an important role by slowing down the binding speed between Rh5 and the receptors of red blood cells, thus making the antibodies to Rh5 more effective [24]. To date, only a few candidate vaccines (such as the RH5.1/AS01B vaccine) have been allowed to be used in phase I/IIa clinical trials. Clearly, more data on the safety, immunogenicity, and efficacy of bloodstage vaccines are still required [25].

\section{Vaccines Targeting the Sexual and Mosquito Stages}

Transmission-blocking vaccines (TBVs) are designed to control the transmission of malaria parasites from human hosts to the mosquito vectors (Figure 2(c)). In the 1950s, Hill et al. [48] first demonstrated the induction of transmissionblocking immunity in chickens by repeated immunization with Plasmodium gallinaceum-infected red blood cell. Since then, significant advances have been made in TBV development. Subsequent studies have shown that antigens expressed later in parasite development after fertilization can be targeted [49]. Sherrard et al. found that TBVs could reduce parasite density in the mosquito salivary glands, thereby enhancing pre-erythrocytic vaccine efficacy [50]. The leading TBV candidates include the Pfs $25, \mathrm{Pfs} 48 / 45$, and Pfs230 which have shown transmission-blocking immunity in model systems in different stages of development. [51]. During the stage of transmission, the T cells usually impact on it. It is reported that the lysate of mature gametocyteinfected RBCs leads to the T-cell proliferation and the expression of IFN- $\gamma$. Moreover, T cells associated with B cells may enhance the immune response during the malaria infection. B-cell knockout mice induced a CD $4^{+} \mathrm{T}$ Th1-like response compared with Th2 cells in the control group, which means that $\mathrm{B}$ cells play a role in the regulation of $\mathrm{CD} 4^{+} \mathrm{T}$ subset responses [36].

4.1. Pfs25. Pfs 25 , a $25 \mathrm{kDa}$ sexual-stage antigen present on the surfaces of $P f$ mosquito-stage gametes and zygotes, has four epidermal growth-like factor structural domains, a large number of cysteine residues, and a complex polymer structure [26, 52]. In 2008, the National Institutes of Health, USA, completed a phase I clinical trial using Pfs 25 as the antigen [27]. Pfs 25 fused to IMX313 and expressed in ChAd63 and MVA viral vectors led to a qualitatively improved antibody response compared with Pfs 25 alone, as well as to significantly higher germinal center responses [28]. Lee et al. [26] reported that Pfs25 expressed in baculovirus and Pichia showed promise for TBV development. Interestingly, Scally et al. [51] reported that six crystal structures of Pfs 25 in complex with the antibodies that were elicited by immunization had good specificity associated with two distinct immunogenic sites on the Pfs 25 protein.

4.2. $P f_{s} 48 / 45$. Pfs $48 / 45$ is expressed on the surfaces of gametocytes (from stage III onwards) and gametes and is bound to the parasite membrane through a GPI anchor. It forms a stable complex with Pfs230, another important TBV candidate. Animal studies have shown that transmissionblocking antibodies can be stimulated by immunization with Pfs48/45 [29]. Human antibodies against Pfs $48 / 45$ and Pfs230 proteins have been investigated in a study in central Ghana [53], where their seroprevalence was found to be 74.7 and $72.8 \%$, respectively [29]. Singhet al. [54] reported that SpyCatcher-R0.6C and SpyCatcher-6C in Pfs48/45-based virus-like particle vaccines were responsible for significantly 
increased antigen immunogenicity when Montanide ISA $720 \mathrm{VG}$ was used as the extrinsic adjuvant.

Current data suggest that in addition to Pfs 25 and Pfs48/ 45 , identifying new target antigens and/or combinations thereof could be a worthwhile strategy for future vaccine research. Structural biology can be used to predict the defensive epitopes, genomics, and proteomics for novel vaccines. Moreover, safe adjuvants that can effectively boost the immune system should be also fully concerned.

\section{Conclusions}

We have described three types of malaria vaccine in this review (Table 1). The completed phase III clinical trial of RTS,S, the most promising vaccine to date, showed that the vaccine's efficacy was limited and that its effect was geographically regional. There is no doubt that more research is needed for malaria vaccine development. Using new tools to identify new target antigens is one of the most important ways in future. Moreover, to increase the immune system response, more kinds of adjuvant systems should be developed to speed up the development of a new generation of malaria vaccine.

In this limited review, we are not covering all approaches to malaria vaccine development, or most of the critically important work on the development of vaccines against $P$. vivax, the second most important cause of malaria. The most difficult part for the development of $P$. vivax vaccines is that the parasite is not tractable to in vitro culture. The lack of a method for the continuous culture of $P$. vivax blood stages makes it difficult to identify synergistic antigens in blood stage. Till now, some groups have successfully established short-term $P$. vivax culture, and more tools for parasite culture are still needed for further development of vaccines against $P$. vivax.

\section{Conflicts of Interest}

The authors confirm no conflicts of interest regarding the publication of this article.

\section{Acknowledgments}

The authors thank Sandra Cheesman, PhD, from Edanz Group (http://www.edanzediting.com/ac) for editing a draft of this manuscript. This work was supported by the R\&D Infrastructure and Facility Development Program of Jilin Province (20170623093) and the Jilin Province Science and Technology Development Plan Project (20180201059YY).

\section{References}

[1] V. Corbel, F. Nosten, K. Thanispong, C. Luxemburger, M. Kongmee, and T. Chareonviriyaphap, "Challenges and prospects for dengue and malaria control in Thailand, Southeast Asia," Trends in Parasitology, vol. 29, no. 1, pp. 623-633, 2013.

[2] P. L. Alonso and M. Tanner, "Public health challenges and prospects for malaria control and elimination," Nature Medicine, vol. 19, no. 2, pp. 150-155, 2013.
[3] B. Mordmüller, G. Surat, H. Lagler et al., "Sterile protection against human malaria by chemoattenuated PfSPZ vaccine," Nature, vol. 542, no. 7642, pp. 445-449, 2017.

[4] M. S. Sissoko, S. A. Healy, A. Katile et al., "Safety and efficacy of PfSPZ vaccine against Plasmodium falciparum via direct venous inoculation in healthy malaria-exposed adults in Mali: a randomised, double-blind phase 1 trial," The Lancet Infectious Diseases, vol. 17, no. 5, pp. 498-509, 2017.

[5] J. C. Pringle, G. Carpi, G. J. Almagro et al., "RTS,S/AS01 malaria vaccine mismatch observed among Plasmodium falciparum isolates from southern and central Africa and globally," Scientific Reports, vol. 8, no. 1, p. 6622, 2018.

[6] Z. Tan, T. Zhou, H. Zheng, Y. Ding, and W. Xu, "Malaria DNA vaccine gp96NTD-CSP elicits both CSP-specific antibody and CD8+ T cell response," Parasitology Research, vol. 114, no. 6, pp. 2333-2339, 2015.

[7] RTS,S Clinical Trials Partnership, S. T. Agnandji, B. Lell et al., "First results of phase 3 trial of RTS,S/AS01 malaria vaccine in African children," The New England journal of medicine, vol. 365, no. 20, pp. 1863-1875, 2011.

[8] RTS,S Clinical Trials Partnership, "Efficacy and safety of RTS,S/AS01 malaria vaccine with or without a booster dose in infants and children in Africa: final results of a phase 3, individually randomised, controlled trial," The Lancet, vol. 386, no. 9988, pp. 31-45, 2015.

[9] K. A. Collins, R. Snaith, M. G. Cottingham, S. C. Gilbert, and A. V.S. Hill, "Enhancing protective immunity to malaria with a highly immunogenic virus-like particle vaccine," Scientific Reports, vol. 7, article 46621, 2017.

[10] D. E. Neafsey, M. Juraska, T. Bedford et al., "Genetic diversity and protective efficacy of the RTS,S/AS01 malaria vaccine," New England Journal of Medicine, vol. 373, no. 21, pp. 2025-2037, 2015.

[11] H. Tinto, W. Otieno, S. Gesase et al., "Long-term incidence of severe malaria following RTS,S/AS01 vaccination in children and infants in Africa: an open-label 3-year extension study of a phase 3 randomised controlled trial," The Lancet Infectious Diseases, vol. 19, no. 8, pp. 821-832, 2019.

[12] J. E. Galen, M. F. Pasetti, S. Tennant, P. Ruiz-Olvera, M. B. Sztein, and M. M. Levine, "Salmonella enterica serovar Typhi live vector vaccines finally come of age," Immunology and Cell Biology, vol. 87, no. 5, pp. 400-412, 2009.

[13] C. Gonzalez, D. Hone, F. R. Noriega et al., "Salmonella typhi vaccine strain CVD 908 expressing the circumsporozoite protein of Plasmodium falciparum: strain construction and safety and immunogenicity in humans," Journal of Infectious Diseases, vol. 169, no. 4, pp. 927-931, 1994.

[14] S. Nazeri, S. Zakeri, A. A. Mehrizi, and N. D. Djadid, "Naturally acquired immune responses to thrombospondinrelated adhesion protein (TRAP) of Plasmodium vivax in patients from areas of unstable malaria transmission," Acta Tropica, vol. 173, pp. 45-54, 2017.

[15] U. Krzych, J. A. Lyon, T. Jareed et al., "T lymphocytes from volunteers immunized with irradiated Plasmodium falciparum sporozoites recognize liver and blood stage malaria antigens," Journal of immunology, vol. 155, no. 8, pp. 40724077, 1995.

[16] L. Schwartz, G. V. Brown, B. Genton, and V. S. Moorthy, "A review of malaria vaccine clinical projects based on the WHO rainbow table," Malaria Journal, vol. 11, no. 1, p. 11, 2012.

[17] E. Prieur and P. Druilhe, "The malaria candidate vaccine liver stage antigen-3 is highly conserved in Plasmodium falciparum isolates from diverse geographical areas," Malaria Journal, vol. 8, p. 247, 2009. 
[18] B. A. Toure, B. L. Perlaza, J. P. Sauzet et al., "Evidence for multiple B- and T-cell epitopes in Plasmodium falciparum liver-stage antigen 3," Infection and immunity, vol. 77, no. 3, pp. 1189-1198, 2009.

[19] J. C. Pizarro, V. Chitarra, D. Verger et al., "Crystal structure of a Fab complex formed with PfMSP1-19, the C-terminal fragment of merozoite surface protein 1 from Plasmodium falciparum: a malaria vaccine candidate," Journal of Molecular Biology, vol. 328, no. 5, pp. 1091-1103, 2003.

[20] C. E. Chitnis, P. Mukherjee, S. Mehta et al., "Correction: phase I clinical trial of a recombinant blood stage vaccine candidate for Plasmodium falciparum malaria based on MSP1 and EBA175," PLoS One, vol. 10, no. 9, Article ID e0137816, 2015.

[21] J. R. Alaro, A. Partridge, K. Miura et al., "A chimeric Plasmodium falciparum merozoite surface protein vaccine induces high titers of parasite growth inhibitory antibodies," Infection and Immunity, vol. 81, no. 10, pp. 3843-3854, 2013.

[22] J. M. Burns, K. Miura, J. Sullivan, C. A. Long, and J. W. Barnwell, "Immunogenicity of a chimeric Plasmodium falciparum merozoite surface protein vaccine in Aotus monkeys," Malaria Journal, vol. 15, p. 159, 2016.

[23] F. Galaway, L. G. Drought, M. Fala et al., "P113 is a merozoite surface protein that binds the $\mathrm{N}$ terminus of Plasmodium falciparum RH5," Nature Communications, vol. 8, no. 3, article 14333, 2007.

[24] G. W. Daniel, Q. Doris, R. Kumarasingha et al., "Human antibodies that slow erythrocyte invasion potentiate malarianeutralizing antibodies," Cell, vol. 178, no. 1, pp. 216-228, 2019.

[25] J. Jin, R. D. Tarrant, E. J. Bolam et al., "Production, quality control, stability, and potency of cGMP-produced Plasmodium falciparum RH5.1 protein vaccine expressed in Drosophila S2 cells," NPJ Vaccines, vol. 3, 2018.

[26] S. M. Lee, C. K. Wu, J. Plieskatt et al., "Assessment of Pfs25 expressed from multiple soluble expression platforms for use as transmission-blocking vaccine candidates," Malaria Journal, vol. 15, no. 1, p. 405, 2016.

[27] Y. Wu, R. D. Ellis, D. Shaffer et al., "Phase 1 trial of malaria transmission blocking vaccine candidates Pfs 25 and Pvs25 formulated with montanide ISA 51," PLoS One, vol. 3, no. 7, Article ID e2636, 2008.

[28] Y. Li, D. B. Leneghan, K. Miura et al., "Enhancing immunogenicity and transmission-blocking activity of malaria vaccines by fusing Pfs 25 to IMX313 multimerization technology," Scientific Reports, vol. 6, article 18848, 2016.

[29] C. S. Jones, T. Luong, M. Hannon et al., "Heterologous expression of the $\mathrm{C}$-terminal antigenic domain of the malaria vaccine candidate Pfs48/45 in the green algae Chlamydomonas reinhardtii," Applied Microbiology and Biotechnology, vol. 97, no. 5, pp. 1987-1995, 2013.

[30] L. R. Geert, L. R. Isabel, C. Frédéric et al., "Evaluation of the immune response to RTS,S/AS01 and RTS,S/AS02 adjuvanted vaccines," Human Vaccines \& Immunotherapeutics, vol. 10, no. 68, pp. 2211-2219, 2014.

[31] S. Chaudhury, J. A. Regules, C. A. Darko et al., "Delayed fractional dose regimen of the RTS,S/AS01 malaria vaccine candidate enhances an IgG4 response that inhibits serum opsonophagocytosis," Scientific Reports, vol. 7, no. 1, p. 7998, 2017.

[32] P. Sun, R. Schwenk, K. White et al., "Protective immunity induced with malaria vaccine, RTS,S, is linked to Plasmodium falciparum circumsporozoite protein-specific CD4+ and CD8+ T cells producing IFN- $\gamma$," The Journal of Immunology, vol. 171, no. 12, pp. 6961-6967, 2003.
[33] V. S. Moorthy, "Immunological mechanisms underlying protection mediated by RTS,S: a review of the available data," Malaria Journal, vol. 8, no. 1, p. 312, 2009.

[34] K. Dundas, M. J. Shears, Y. Sun et al., "Alpha-v-containing integrins are host receptors for the Plasmodium falciparum sporozoite surface protein, TRAP," Proceedings of the $\mathrm{Na}$ tional Academy of Sciences, vol. 115, no. 17, pp. 4477-4482, 2018.

[35] K. J. Ewer, G. A. O’Hara, C. J. Duncan et al., "Protective CD8+ T-cell immunity to human malaria induced by chimpanzee adenovirus-MVA immunisation," Nature Communications, vol. 4, p. 2836, 2013.

[36] S. L. Hoffmana, J. Vekemansb, T. L. Richiea, and P. E. Duffyc, "The march toward malaria vaccines," American Journal of Preventive Medicine, vol. 49, no. 6, pp. 319-333, 2015.

[37] S. Capone, A. Reyes-Sandoval, M. Naddeo et al., "Immune responses against a liver-stage malaria antigen induced by simian adenoviral vector AdCh63 and MVA prime-boost immunisation in non-human primates," Vaccine, vol. 29, no. 2, pp. 256-265, 2010.

[38] S. H. Hodgson, K. J. Ewer, C. M. Bliss et al., "Evaluation of the efficacy of Chad63-MVA vectored vaccines expressing circumsporozoite protein and ME-TRAP against controlled human malaria infection in malaria-naive individuals," Journal of Infectious Diseases, vol. 211, no. 7, pp. 1076-1086, 2015.

[39] O. G. Gomez, M. F. Pasetti, A. Santiago, M. B. Sztein, S. L. Hoffman, and M. M. Levine, "Expression, extracellular secretion, and immunogenicity of the Plasmodium falciparum sporozoite surface protein 2 in Salmonella vaccine strains," Infection and immunity, vol. 69, no. 2, pp. 1192-1198, 2001.

[40] W. S. Nicoll, J. B. Sacci, C. Rodolfo et al., "Plasmodium falciparum liver stage antigen-1 is cross-linked by tissue transglutaminase," Malaria Journal, vol. 10, no. 1, p. 14, 2011.

[41] J. E. Epstein, K. M. Paolino, T. L. Richie et al., "Protection against Plasmodium falciparum malaria by PfSPZ vaccine," JCI Insight, vol. 2, no. 1, article e89154, 2017.

[42] J. E. Epstein, K. Tewari, K. E. Lyke et al., "Live attenuated malaria vaccine designed to protect through hepatic CD8+ T cell immunity," Science, vol. 334 , no. 6055 , pp. 475-480, 2011.

[43] S.-I. Inoue, M. Niikura, S. Takeo et al., "Enhancement of dendritic cell activation via CD40 ligand-expressing $\gamma \delta$ T cells is responsible for protective immunity to Plasmodium parasites," Proceedings of the National Academy of Sciences, vol. 109, no. 30, pp. 12129-12134, 2012.

[44] A. L. Goodman and S. J. Draper, "Blood-stage malaria vaccines-recent progress and future challenges," Annals of Tropical Medicine \& Parasitology, vol. 104, no. 3, pp. 189-211, 2010.

[45] S. H. Sheehy, C. J. Duncan, S. C. Elias et al., "Chad63-MVAvectored blood-stage malaria vaccines targeting MSP1 and AMA1: assessment of efficacy against mosquito bite challenge in humans," Molecular Therapy, vol. 20, no. 12, pp. 23552368, 2012.

[46] B. Genton, F. Al-Yaman, I. Betuela et al., "Safety and immunogenicity of a three-component blood-stage malaria vaccine (MSP1, MSP2, RESA) against Plasmodium falciparum in Papua New Guinean children," Vaccine, vol. 22, no. 1, pp. 30-41, 2003.

[47] S. L. Takala, D. Coulibaly, M. A. Thera et al., "Dynamics of polymorphism in a malaria vaccine antigen at a vaccinetesting site in Mali," PLoS Medicine, vol. 4, no. 3, p. 93, 2007. 
[48] C. A. Hill, F. C. Kafatos, S. K. Stansfield, and F. H. Collins, "Arthropod-borne diseases: vector control in the genomics era," Nature Reviews Microbiology, vol. 3, no. 3, pp. 262-268, 2005.

[49] C. A. Long and F. Zavala, "Malaria vaccines and human immune responses," Current Opinion in Microbiology, vol. 32, pp. 96-102, 2016.

[50] E. Sherrard-Smith, K. A. Sala, M. Betancourt et al., "Synergy in anti-malarial pre-erythrocytic and transmission-blocking antibodies is achieved by reducing parasite density," eLife, vol. 7, article e35213, 2018.

[51] S. W. Scally, B. McLeod, A. Bosch et al., "Molecular definition of multiple sites of antibody inhibition of malaria transmission-blocking vaccine antigen Pfs25," Nature Communications, vol. 8, no. 1, p. 1568, 2017.

[52] D. C. Kaslow, I. A. Quakyi, C. Syin et al., “A vaccine candidate from the sexual stage of human malaria that contains EGFlike domains," Nature, vol. 333, no. 6168, pp. 74-76, 1988.

[53] F. K. Acquah, E. K. Obboh, K. Asare et al., "Antibody responses to two new Lactococcus lactis-produced recombinant Pfs $48 / 45$ and Pfs 230 proteins increase with age in malaria patients living in the Central Region of Ghana," Malaria Journal, vol. 16, no. 1, p. 306, 2017.

[54] S. K. Singh, S. Thrane, C. M. Janitzek et al., "Improving the malaria transmission-blocking activity of a Plasmodium falciparum 48/45 based vaccine antigen by SpyTag/SpyCatcher mediated virus-like display," Vaccine, vol. 35, no. 30, pp. 3726-3732, 2017. 


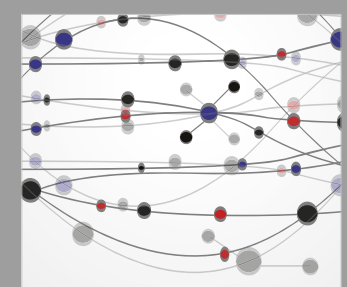

The Scientific World Journal
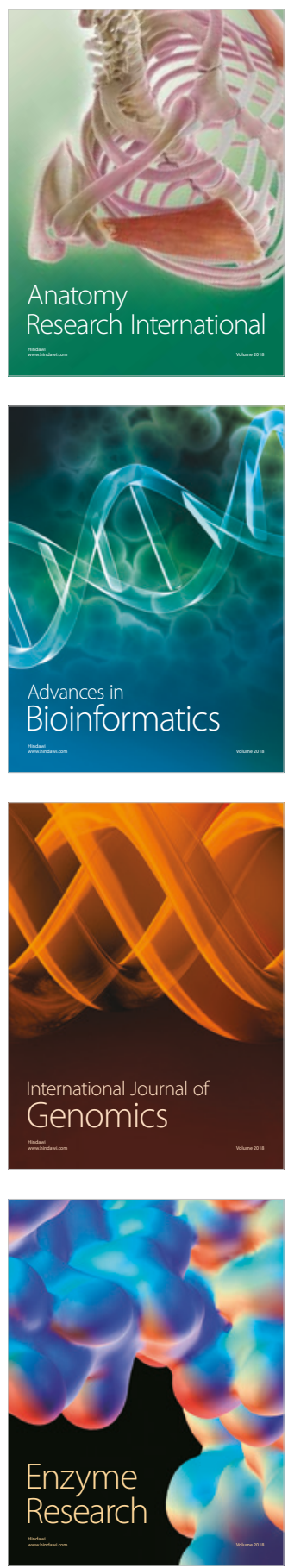
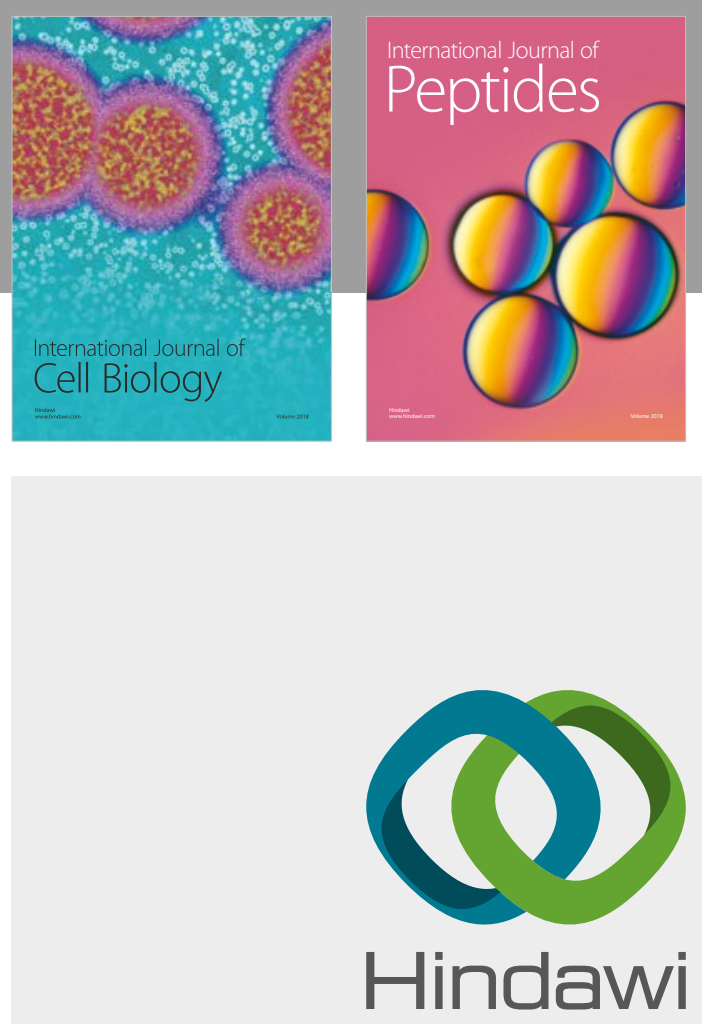

Submit your manuscripts at

www.hindawi.com
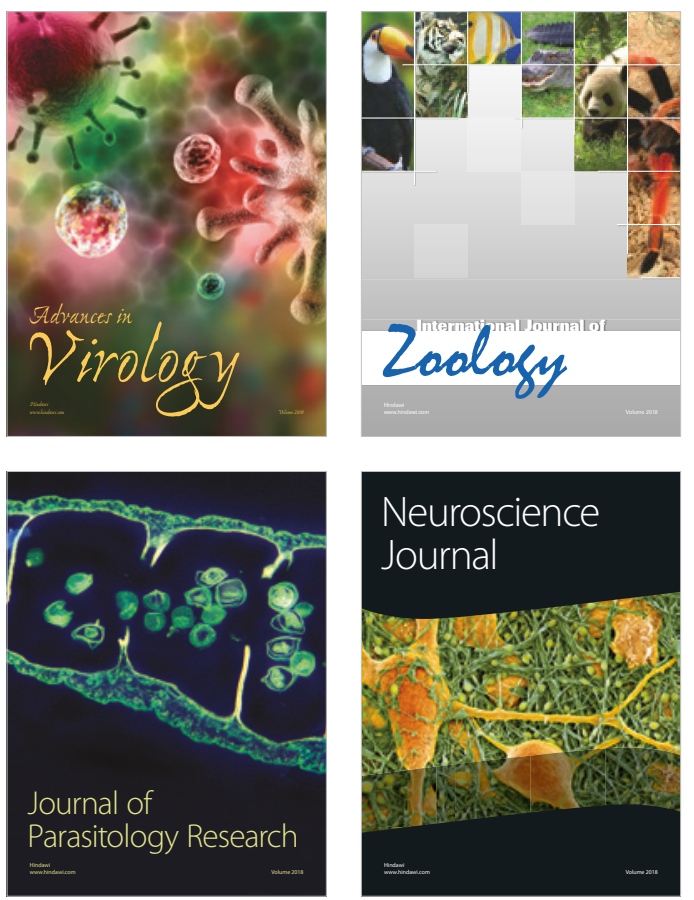
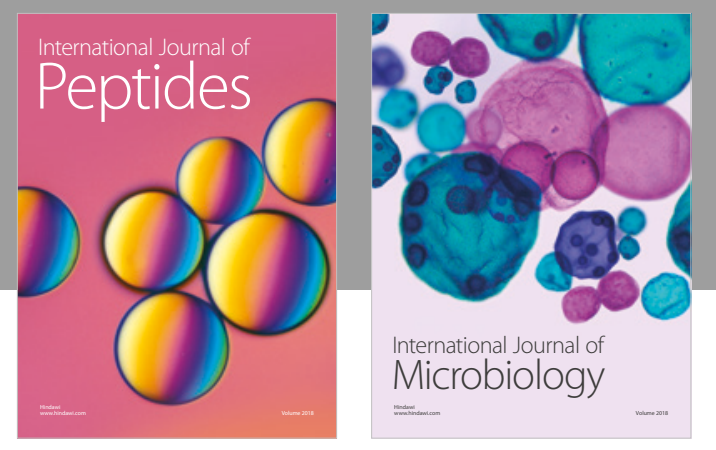

nternational Journal of Microbiology
Journal of
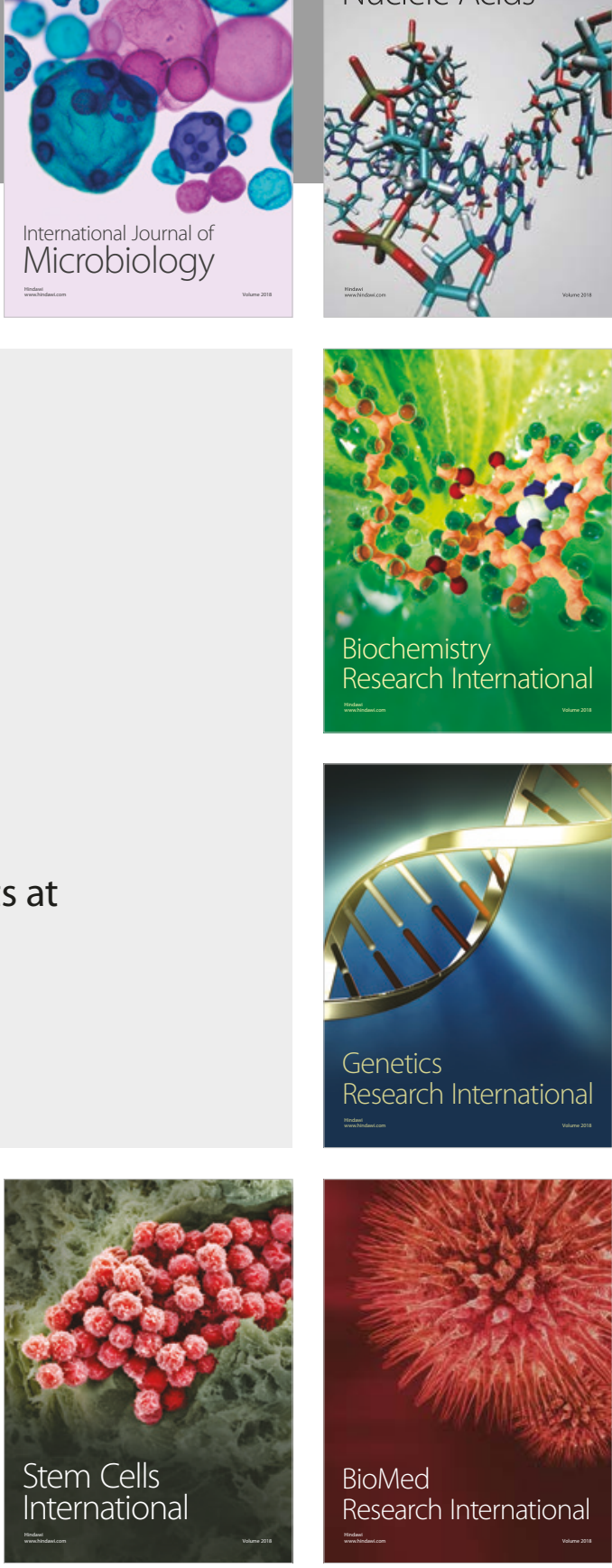
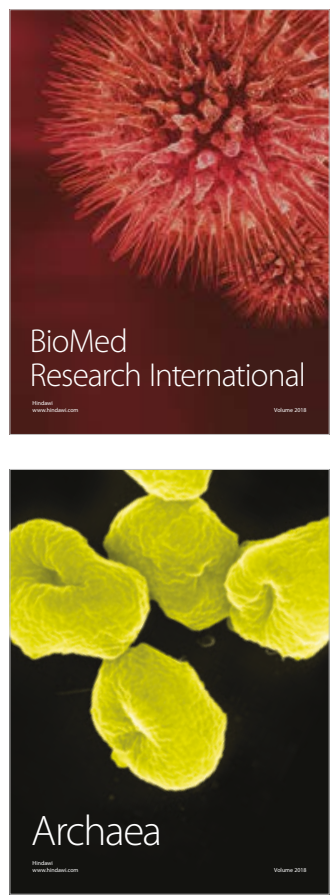